\title{
DRONE-BASED FLUORESCENCE LIDAR SYSTEMS FOR VEGETATION AND MARINE ENVIRONMENT MONITORING
}

\author{
Zheng Duan ${ }^{1}$, Ying Li ${ }^{1}$, Xun Wang ${ }^{1}$, Jinlei Wang ${ }^{1}$, Mikkel Brydegaard ${ }^{2}$, \\ Guangyu Zhao ${ }^{1}$ and Sune Svanberg ${ }^{1,2^{*}}$ \\ ${ }^{1}$ Center for Optical and Electromagnetic Research, South China Academy of Advanced Optoelectronics, \\ South China Normal University, University City Campus, Guangzhou 510006, China \\ ${ }^{2}$ Department of Physics, Lund University, SE 22100 Lund, Sweden \\ *Email: sune.svanberg@fysik.lth.se
}

\begin{abstract}
We have developed two different types of dronebased fluorescence lidar systems for vegetation and marine environment monitoring, both based on violet $\mathrm{CW}$ diode lasers. An inelastic hyperspectral Scheimpflug lidar system was used for vegetation profiling combined with fluorescence spectral recordings. A light-weight fluorosensor set for fixed-height recordings was employed for monitoring of marine environments, featuring water Raman signals, algal chlorophyll and strong oil spill fluorescence.
\end{abstract}

\section{INTRODUCTION}

In the study of vegetation in agriculture and forestry, chlorophyll, responsible for photosynthesis, is an essential indicator to reflect the growing conditions of vegetation. Chemical methods to monitor chlorophyll inside vegetation, e.g., extraction by acetone [1] are time-consuming and only available for laboratory quantification. Optical methods to monitor vegetation have merits of fast-response, non-invasive detection and high-sensitivity and are adapted to meet the need for large-scale monitoring and remote sensing. Laser-induced fluorescence spectroscopy (LIF) techniques are highly suitable for real-time, in-situ and non-invasive monitoring of vegetation status. When a short-wavelength excitation laser beam is impinging on vegetation, chlorophyll will efficiently absorb the radiation and re-emit fluorescence at longer wavelengths, which provides a finger-print characteristic of chlorophyll [2]. Combined with remote sensing techniques, the laser-induced fluorescence technique was applied to early remote monitoring studies of vegetation, e.g., spruce, maize and maple [3]; sugar beets [4] and corn [5]. In the past years we constructed a flexible mobile pulsed laser lidar system for environmental monitoring [6] and we reported fluorescence lidar applications for hybrid maize and hybrid rice $[7,8]$. However, while perfect for monitoring of, e.g., atmospheric atomic mercury [9], such a system is complex and expensive to operate when it comes to fluorescence monitoring, which is much less demanding [10]. The introduction of a compact inelastic hyperspectral Scheimpflug lidar system [11], makes it possible to realize simultaneous spatial and spectral information of distributed targets. Based on a commercial drone platform, we have developed a miniaturized hyperspectral lidar system for profiling the vegetation structure [12].

\section{METHODOLOGY}

\subsection{Drone-based inelastic hyperspectral Scheimpflug lidar for vegetation monitoring}

The setup used in our vegetation studies can be seen in Fig. 1. A commercial drone (DJI M600 Pro) with a maximum lifting capacity of $6.2 \mathrm{~kg}$ is utilized to carry the hyperspectral Scheimpflug lidar system. The excitation laser is a $1.5 \mathrm{~W} 445$ $\mathrm{nm}$ Nichia diode laser with an adjustable-focus objective from Computar. A common industrial control computer is used for data capturing and storage. When the backscattered light from the objects is captured by the receiving telescope, the light, after beam redirection by a folding mirror, will pass through a slit with a width of $100 \mu \mathrm{m}$. A prism-grating-prism structure is used to disperse the light. After collimation by lenses, the light will be distributed on the imaging plane of an area CCD. A combination of the spatial and spectral information will be obtained in every frame. 


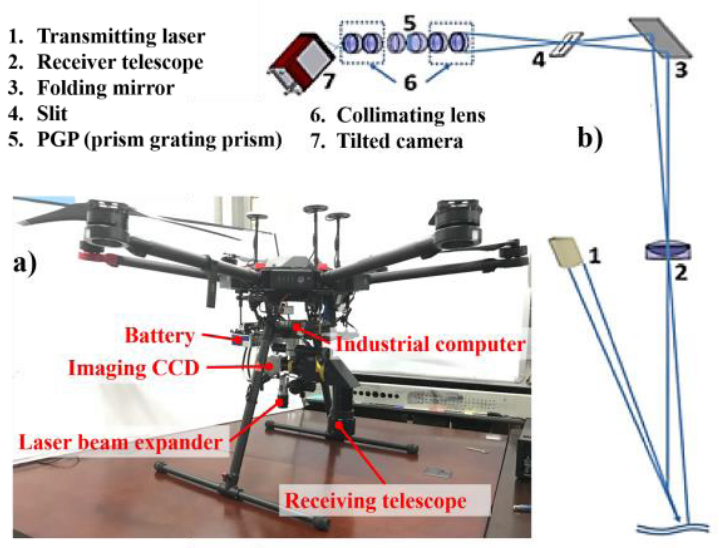

Fig. 1. a) Drone-based inelastic hyperspectral Scheimpflug lidar for vegetation monitoring; b) Schematic of the lidar system, modified from [12].

\subsection{Drone-based fluorosensor for marine environment monitoring}

The fluorosensor is equipped with a $1 \mathrm{~W}, 412 \mathrm{~nm}$ diode laser (Xinrui, Model 412) and uses a compact spectrometer (USB4000 Ocean Optics) as the detector as seen in Fig. 2. A $425 \mathrm{~nm}$ longpass filter (Edmund Optics \#84-742) is used to cut off the excitation laser radiation. By tilting the laser, which is mounted non-coaxially next to the $50 \mathrm{~mm}$ diameter telescope with $200 \mathrm{~mm}$ focus, the measurement range is set to about $10 \mathrm{~m}$. The laser beam is transmitted from the diode laser using a small collimator, and a telescope with a filter mounted after the objective is used to collect the fluorescence from the target. The laser can be directed at a proper angle to overlap with the telescope field-of-view as tuned according to the distance to the target. After the deflection from the mirror, the fluorescence is focused into the spectrometer and the data will be stored in an industrial control computer.

\section{RESULTS}

\subsection{Vegetation monitoring}

As a demonstration, we have monitored two trees behind our laboratory on the University City campus of South China Normal University with
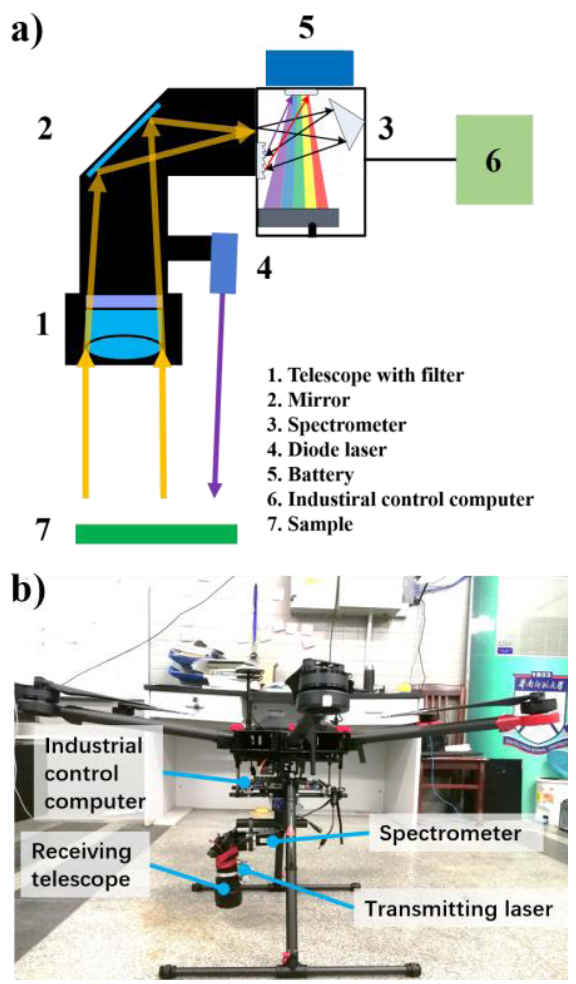

Fig. 2. a) Schematic of the fluorosensor; b) Drone-based fluorosensor for marine environment monitoring.

our inelastic hyperspectral Scheimpflug lidar based on a drone. As shown in Fig. 3, through integrating GPS (global positioning information) and INS (inertia navigation system) data with recorded frames, we can reconstruct these two trees with 3D point clouds with color coding of the spectral information. The fluorescence ratio between $690 \mathrm{~nm}$ and $730 \mathrm{~nm}$ was calculated as a relative measure of the concentration of chlorophyll in the vegetation. The yellow spots indicate the elastic signals without spectral information. Light from the road lamp constituting background noise could be suppressed by modulating the laser output.

\subsection{Marine environment monitoring}

The drone-based fluorosensor system is utilized to perform marine environment monitoring on the Zhujiang (Pearl) river, flowing with many branches through the city of Guangzhou. Two 90$\mathrm{cm}$-diameter floating containers were positioned on the surface of the river as artificial targets. One was filled with 1 litre of pure engine oil, resulting in layer thickness of typically 1-2 mm, and the other one was filled with 10 litres of a dilute solution of Rhodamine 610 dye with the 

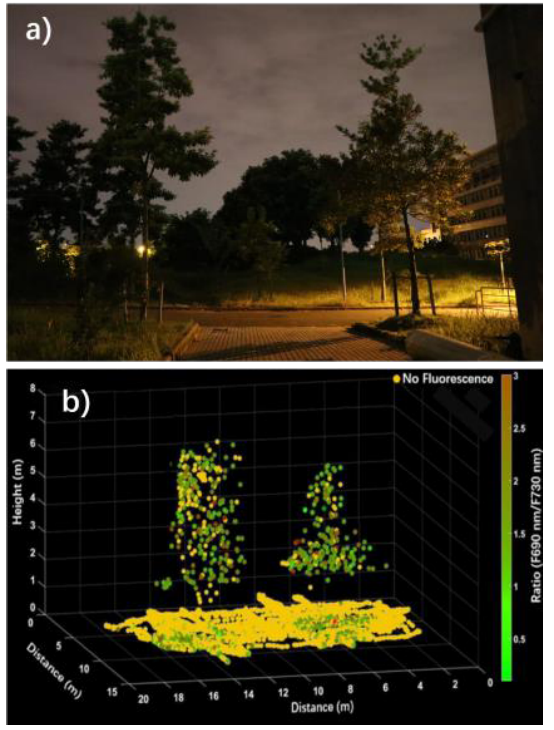

Fig. 3. a) Photo of two trees as targets; b) Point cloud figure of two trees with spectral information captured by the drone lidar; Color map coding corresponding to the ratio of chlorophyll fluorescence (F690/F730) is employed; modified from [12].

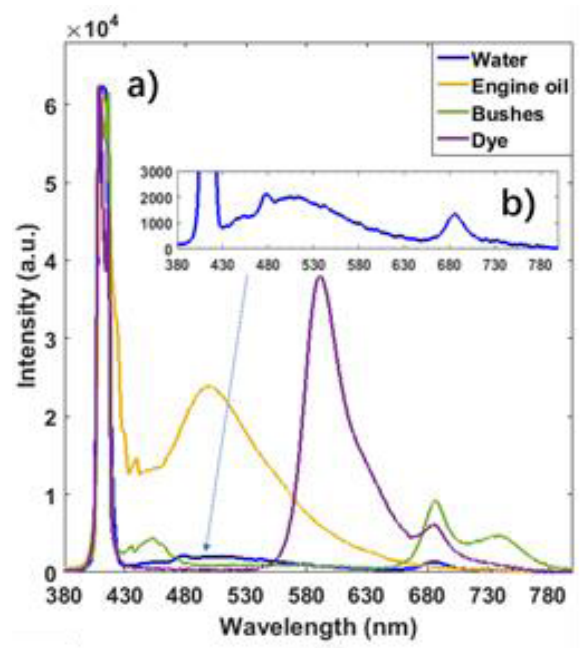

c)

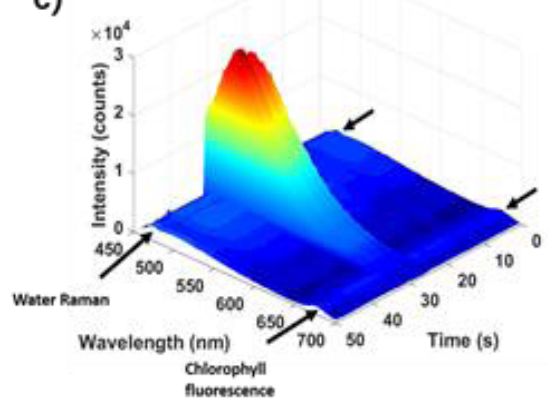

Fig. 4. a) Spectra captured by the drone-based flurosensor over the Zhujiang river; b) Zoom-in spectrum of natural Zhujiang river; c) Result of a linear scan of an oil-filled floating container on Zhujiang river. The exposure time of the spectrometer was set to $0.5 \mathrm{~s}$; modified from [17]. concentration of $0.03 \mathrm{~g} / 1$, resulting in a $1-2 \mathrm{~cm}$ layer thickness. The dye is dissolved in natural Zhujiang river water. With the drone scanning along a setup flight route covering the artificial targets, the measurements are illustrated in Fig. 4 a) and b). Four typical spectra recorded by the flurosensor are presented. The characteristic fluorescence peak of oil at $500 \mathrm{~nm}$ and dye fluorescence peak at $580 \mathrm{~nm}$ can be observed. Vegetation on the riverbank was also covered, and the strong chlorophyll characteristic peaks at 685 $\mathrm{nm}$ and $745 \mathrm{~nm}$ are recorded. In the zoom-in spectrum of the natural Zhujiang water, strong dissolved organic matter (DOM) fluorescence and algal chlorophyll signals are shown, as well as the water Raman peak at $480 \mathrm{~nm}$. A linear scan of the oil-filled container with a continuous recording of $50 \mathrm{~s}$ is presented in Fig. $4 \mathrm{c}$ ). High sensitivity of the fluorosensor towards oil spills can be noticed, as contrasted to the background water Raman, algal chlorophyll and DOM signals.

\section{ACKNOWLEDGEMENTS}

This work was supported by the National Science Foundation of China under Grant No. 61705069 and the Chinese Ministry of Science and Technology through the National Key Research and Development Program of China under Grant No. 2018YFC1407503 and the Special Funds Program for the Cultivation of Guangdong College Students' Scientific and Technologicaol Innovation Climbing Project under Grant No. pdjha0127.

\section{REFERENCES}

[1] H. K. Lichtenthaler, Methods in Enzymology 148: 350-382 (1987)

[2] H. K. Lichtenthaler, et al. CRC Critical Reviews in Analytical Chemistry 19: S29-S58 (1988)

[3] S. Svanberg, Physica. Scripta T58: 79-85 (1995)

[4] Z. G. Cerovic, et al. Biochimica et Biophysica Acta (BBA) - Bioenergetics 1188: 58-68 (1994)

[5] B. Xu, et al. Journal of Remote Sensing 7(S1): 5159 (1992)

[6] G. Y. Zhao, et al. Applied Optics 56(5): 1506-1516 (2017)

[7] G. Y. Zhao, et al. Applied Optics 55(19): 52735279 (2016)

[8] Z. Duan, et al. Applied Optics 57(13): 3481-3487 (2018)

[9] M. Lian, et al. Environmental Pollution 240: 353358 (2018) 
[10] S. Svanberg, in Laser Remote Sensing (Eds: T. Fujii, T. Fukuchi), CRC Press, Boca Raton, FL 2005, pp 433-467

[11] G. Y. Zhao, et al. Lasers \& Photonics Reviews 10(5): 807-813 (2016)

[12] X. Wang, et al. Applied Physics B 124: 207 (2018)

[13] S. Svanberg, in Optoelectronics for Environmental Science (Eds: S. Martellucci, A. N. Chester), Springer, Berlin Heidelberg 1990, pp 15-27

[14] F. E. Hoge, et al. Applied Optics 19(19): 32693281 (1980)

[15] M. Lenon, et al. European Oceans 2005(2): 848-

853 (2005)

[16] A. Pisao, et al. Remote Sensing 7(1): 1112-1134 (2015)

[17] Z. Duan, et al. Applied Physics B 125(11): 108 (2019) 